\title{
Pigeons' memory for sequences of light flashes when gap duration is an unreliable discriminative cue
}

\author{
Dwayne Keough, Angelo Santi, and Patrick Van Rooyen \\ Wilfrid Laurier University, Waterloo, Ontario, Canada
}

\begin{abstract}
In Experiment 1, pigeons were trained with a 1-sec dark and a 1-sec houselight-illuminated delay interval to discriminate between sequences of two and four flashes of light (feeder illumination). The sequences could be discriminated on the basis of the number of flashes, the number of gaps, or the duration of the gap between flashes. A choose-few bias was obtained at extended dark delays, but not at extended illuminated delays. Pigeons appeared to confuse long dark delays with the longer gap between flashes on few-sample trials. In Experiment 2, additional sample sequences were included that made gap duration an unreliable cue for discriminating between the few and many samples. A significant choose-many bias was obtained at extended dark delay intervals, but no biased forgetting was found at extended illuminated delays. The pigeons appeared to discriminate light flash sequences by relying on multiple temporal features of a sequence rather than using an event switch to count flashes. The biased-forgetting effects observed appear to be due to instructional ambiguity that results from the similarity of the delay interval to features of the flash sequences.
\end{abstract}

In studies of memory for event duration, pigeons are trained using a delayed symbolic matching-to-sample (DSMTS) procedure to discriminate the duration of a sample stimulus (e.g., 2 vs. 8 sec of houselight illumination). A peck to one comparison stimulus (e.g., red) is reinforced if the sample duration is short, and a peck to the other comparison stimulus (e.g., green) is reinforced if the sample duration is long. Working memory is tested by inserting a delay interval between the end of sample presentation and the onset of comparison stimuli. As demonstrated initially by Spetch and Wilkie (1983), the retention function for the long sample duration drops below chance, whereas the retention function for the short sample duration remains well above chance over delays of 10 and $20 \mathrm{sec}$. Spetch and Wilkie referred to this result as the choose-short effect, because pigeons show a bias to peck the comparison stimulus associated with the short sample as the delay interval is extended beyond baseline training. This result has been reported in a large number of studies (Fetterman, 1995; Gaitan \& Wixted, 2000; Grant, 1993, 2006; Grant \& Kelly, 1996, 1998; Grant \& Spetch, 1991, 1993, 1994; Grant, Spetch, \& Kelly, 1997; Kelly \& Spetch, 2000; Kraemer, Mazmanian, \& Roberts, 1985; Santi, Bridson, \& Ducharme, 1993; Santi, Ducharme, \& Bridson, 1992; Sherburne, Zentall, \& Kaiser, 1998; Spetch, 1987; Spetch \& Rusak, 1989, 1992).

Memory for number of events has also been studied by training pigeons in a DSMTS procedure (Fetterman, 2000; Fetterman \& MacEwen, 1989; Hope \& Santi, 2004; Roberts, Macuda, \& Brodbeck, 1995; Santi \& Hope,
2001). In the studies by Roberts et al. and Santi and Hope, pigeons were presented with sample stimuli consisting of two flashes of light in $4 \mathrm{sec}(2 \mathrm{f} / 4)$ or eight flashes of light in $4 \sec (8 \mathrm{f} / 4)$. These sample sequences will be referred to as few and many in the present paper. A peck to one comparison stimulus (e.g., red) was reinforced if the sample number was few, and a peck to the other comparison stimulus (e.g., green) was reinforced if the sample number was many. Pigeons' memory for number was assessed by inserting delay intervals of varying length between termination of the sample sequence and the onset of the comparison stimuli. At delays greater than the baseline training delay, pigeons responded with higher accuracy following the few sample, whereas response accuracy following the many sample dropped well below chance (Roberts et al., 1995; Santi \& Hope, 2001). This result is referred to as the choose-few effect because pigeons are biased to respond to the comparison stimulus that was correct for the smaller number sample as the delay interval is extended beyond baseline training.

The choose-few effect for number and the choose-short effect for time have been viewed as support for the hypothesis that there is a common mechanism for the processing of temporal and numerical information in pigeons (Roberts \& Mitchell, 1994). The common mechanism is the accumulation of pulses generated by an internal clock as outlined in the mode-control version of the scalar timing theory proposed by Meck and Church (1983). The modecontrol model predicts that timing and counting are both performed by an accumulator that monitors the number

A.Santi, asanti@wlu.ca 
of pulses transmitted from a pacemaker. It was suggested that in order for animals to simultaneously process time and number, separate switches and accumulators operate in a run mode or in an event mode to process time and number, respectively (Roberts et al., 1995). If time and number are processed by the same mechanism, then pulse counts that diminish over an increased delay interval in the timing paradigm should similarly diminish in the number paradigm. Thus, the choose-few effect and the chooseshort effect have been attributed to pigeons' coding event duration and number in a retrospective, analogical form, with response biases during delay testing occurring because the analogical representation foreshortens (time) or shrinks (number) during the delay interval (Roberts et al., 1995). In accord with this account, both the choose-few effect and the choose-short effect have been shown to depend on the baseline training delay. If a constant delay interval (e.g., 5 or $10 \mathrm{sec}$ ) is used during baseline training in either a number (Santi \& Hope, 2001) or a duration (Spetch, 1987) discrimination, then the introduction of shorter delay intervals during testing results in a choosemany effect for number samples and a choose-long effect for duration samples.

Hope and Santi (2004) examined pigeons' memory for number of feeder light flashes, varying the ambient chamber illumination during the intertrial interval (ITI) and the delay interval. In Experiment 2, they found that pigeons tested at shorter or longer illuminated delays after having received baseline training with a 5 -sec illuminated delay interval produced patterns of response biases dissimilar to those previously obtained with dark delays. Specifically, a choose-few bias resulted at delays shorter than baseline training, and a choose-many bias resulted at delays longer than baseline training. The presence of the illuminated houselight during the delay interval appeared to increase the perceived number of events signaled by flashes of feeder light. Hope and Santi suggested that the pigeons may have cumulatively timed each flash of feeder light illumination during an individual trial and then continued to time the duration of houselight illumination throughout the delay interval. In support of this interpretation, Hope and Santi found that when the duration of the second flash on few-sample trials was increased, accuracy fell to chance levels. Thus, pigeons may have coded the number samples by timing and summing flash duration, as opposed to using an event switch to count the number of feeder light flashes. If this interpretation is correct, then the studies by Roberts et al. (1995), Santi and Hope (2001), and Hope and Santi (2004) were not studies examining memory for number, but studies of memory for event duration. Table 1 identifies the temporal and numerical features of the few and many samples used in previous studies of memory for number in the pigeon. Neither the total sequence duration nor the flash duration could have served as a reliable discriminative cue. However, several cues, in addition to the number of flashes, were available. These additional cues include the number of gaps, the sum of the flash durations, the gap between the flashes, and the sum of the gap durations.

Santi, Lellwitz, and Gagne (2006) recently examined memory for number of feeder light flashes using a procedure that controlled for the sum of the flash durations in addition to the duration of individual flashes and the total duration of a sequence. The two sets of sample sequences used by Santi et al. (2006) are shown in the top half of Table 2, where they are identified as gap duration varying (GDV) samples. For both sets, two flashes in $4 \mathrm{sec}$ $(2 \mathrm{f} / 4)$ represented the few sample and four flashes in $4 \mathrm{sec}$ (4f/4) represented the many sample. Accurate responding to these sequences could not be based on the total duration of a sequence (constant $4 \mathrm{sec}$ ), the duration of an individual flash (both Few1 and Many2 have 400-msec flashes), the sum of the flash durations (the flash durations sum to 800 and $1,600 \mathrm{msec}$ for both few and many samples), or the sum of the gap durations (the gaps sum to approximately 3,200 and 2,400 msec for both few and many samples). Other than number of flashes or number of gaps, the only reliable temporal cue remaining that could serve as a discriminative cue was the gap duration. Hence, these samples are referred to as "gap duration varying samples" in Table 2, because the gap duration differed by at least $1,333 \mathrm{msec}$ between the few samples and the many samples. Santi et al. (2006) found that when the delay interval was dark, the retention functions for these samples exhibited a choose-many bias at the 1-sec delay and a choose-few bias at the 4- and 8-sec delays. These effects were observed regardless of whether the pigeons had been trained with a dark ITI (Group Dark) or an illuminated ITI (Group Light). However, when the delay interval was illuminated by the houselight, there was a slight choose-few effect for Group Dark, and no response bias effect for Group Light. The response bias patterns observed appeared to have been due to instructional ambiguity (Dorrance, Kaiser, \& Zentall, 2000; Sherburne et al., 1998; Zentall, 1997, 1999, 2005). Although the term instructional ambiguity has most often been used to refer to similarity between the delay interval and the ITI, in this case the ambiguity comes from the similarity of the delay interval to the gap between light flashes. That is, the short dark delay was confused with the brief

Table 1

Temporal and Numerical Features of Samples Used in Previous Studies of Memory for Number of Light Flashes

\begin{tabular}{ccccccccc}
\hline & $\begin{array}{c}\text { Total } \\
\text { Sequence } \\
\text { Duration }\end{array}$ & $\begin{array}{c}\text { Flash } \\
\text { Duration }\end{array}$ & $\begin{array}{c}\text { No. of } \\
\text { Flashes }\end{array}$ & $\begin{array}{c}\text { No. of } \\
\text { Gaps }\end{array}$ & $\begin{array}{c}\text { Sum of } \\
\text { Flashes }\end{array}$ & $\begin{array}{c}\text { Gap } \\
\text { Between } \\
\text { Flashes }\end{array}$ & $\begin{array}{c}\text { Sum of } \\
\text { Gap } \\
\text { Durations }\end{array}$ & Response \\
\hline $2 \mathrm{f} / 4$ & 4,000 & 200 & 2 & 1 & 400 & 3,600 & 3,600 & red \\
$8 \mathrm{f} / 4$ & 4,000 & 200 & 8 & 7 & 1,600 & 343 & 2,401 & green \\
\hline
\end{tabular}

Note $-2 \mathrm{f} / 4=$ two flashes $/ 4 \mathrm{sec}$. Durations and gaps are in milliseconds. 
Table 2

Temporal and Numerical Features of the Samples Used in Experiments 1 and 2

\begin{tabular}{|c|c|c|c|c|c|c|c|c|}
\hline Sample Type & $\begin{array}{c}\text { Total } \\
\text { Sequence } \\
\text { Duration }\end{array}$ & $\begin{array}{c}\text { Flash } \\
\text { Duration }\end{array}$ & $\begin{array}{c}\text { Sum of } \\
\text { Flash } \\
\text { Durations }\end{array}$ & $\begin{array}{c}\text { Sum of } \\
\text { Gap } \\
\text { Durations }\end{array}$ & $\begin{array}{l}\text { No. of } \\
\text { Flashes }\end{array}$ & $\begin{array}{c}\text { No. of } \\
\text { Gaps }\end{array}$ & $\begin{array}{c}\text { Gap } \\
\text { Between } \\
\text { Flashes }\end{array}$ & Response \\
\hline \multicolumn{9}{|l|}{ GDV Samples } \\
\hline Few1 (2f/4) & 4,000 & 400 & 800 & 3,200 & 2 & 1 & 3,200 & red \\
\hline Few2 (2f/4) & 4,000 & 800 & 1,600 & 2,400 & 2 & 1 & 2,400 & red \\
\hline Many1 (4f/4) & 4,000 & 200 & 800 & 3,201 & 4 & 3 & 1,067 & green \\
\hline Many2 (4f/4) & 4,000 & 400 & 1,600 & 2,400 & 4 & 3 & 800 & green \\
\hline \multicolumn{9}{|l|}{ SDV Samples } \\
\hline Few1 (2f/1.9) & 1,867 & 400 & 800 & 1,067 & 2 & 1 & 1,067 & red \\
\hline Few2 (2f/2.4) & 2,400 & 800 & 1,600 & 800 & 2 & 1 & 800 & red \\
\hline Many1 (4f/10.4) & 10,400 & 200 & 800 & 9,600 & 4 & 3 & 3,200 & green \\
\hline Many2 (4f/8.8) & 8,800 & 400 & 1,600 & 7,200 & 4 & 3 & 2,400 & green \\
\hline
\end{tabular}

Note-Only GDV (gap duration varying) samples were used in Experiment 1. Both GDV and SDV (sequence duration varying) samples were used in Experiment 2. $2 \mathrm{f} / 4=$ two flashes $/ 4 \mathrm{sec}$. Durations and gaps are in milliseconds.

dark gap between flashes of the many samples, resulting in a response to the comparison stimulus correct for many samples. Longer dark delay intervals were confused with the longer dark gap between flashes of the few samples, resulting in a response to the comparison stimulus that was correct for few samples. Illuminated delays were less similar to the dark gaps between flashes, so this reduced instructional ambiguity and resulted in more symmetrical retention functions.

The purpose of the following experiments was to provide additional information on memory for light flash sequences in pigeons. The first experiment replicated Santi et al.'s (2006) finding of a choose-few effect at extended dark delays, but no biased forgetting at extended illuminated delays. The second experiment examined memory for light flash sequences with samples that controlled for the gap between flashes so that the only reliable cue for responding to comparisons was the number of flashes or the number of gaps.

\section{EXPERIMENT 1}

In Experiment 3 of Santi et al. (2006), pigeons received baseline training with a constant 1 -sec dark delay interval in order to prevent the pigeons from responding following this delay interval as if it was the short dark gap between light flashes on the many-sample trials. Pigeons trained with an illuminated ITI were not able to perform accurately with a constant $1-$ sec dark delay. However, the pigeons trained with a dark ITI were able to perform accurately with a constant 1-sec dark delay, and as a result they were delay tested. For these pigeons, training eliminated the choose-many bias at the 1-sec dark delay; however, at extended dark delay intervals, a large choose-few effect was still observed. With illuminated delays, there was a much smaller choose-few bias that remained constant across delays of greater than $1 \mathrm{sec}$. Santi et al. (2006) conjectured that this may have occurred because trials with illuminated delays were treated as if they were trials with few or no gaps. Because multiple gaps occurred only on many-sample trials, there may have been a slight bias to respond to the few-associated comparison when the delay was illuminated. To determine how reliable the modest choose-few effect is with illuminated delays, in Experiment 1 of the present study the pigeons previously used in Santi et al. (2006) were all trained with a dark ITI to discriminate sequences of light flashes with a 1-sec dark and a 1-sec illuminated delay interval. Delay testing at shorter (0-sec) and longer (2-, 4-, and 8-sec) dark and illuminated delays was conducted. It was predicted that testing at extended dark delays would replicate the robust choose-few effect previously reported, as a result of the ambiguity created by the similarity of the delay interval to the gap between light flashes. Making a prediction for retention functions with illuminated delays was less clear. If illuminated delays substantially reduced the similarity of the delay interval to the gap between light flashes, there should be no response bias and the retention functions should be symmetrical.

\section{Method}

Subjects. Subjects were 8 adult Silver King pigeons, maintained at approximately $80 \%$ of their free-feeding weights and housed individually with constant access to grit and water. Postsession feedings of Purina Pigeon Chow were provided to maintain the desired weights for testing. The colony room was illuminated on a 12:12-h light:dark cycle by fluorescent light turned on at 6:00 a.m. daily. Testing occurred 5 days a week, Monday through Friday, between 8:30 a.m. and 1:00 p.m. These pigeons had previously been trained to discriminate sequences of light flashes that varied in the number of flashes, the number of gaps, and the duration of the gap between flashes (Experiments 2 and 3 in Santi et al., 2006).

Apparatus. Four Coulbourn modular operant test cages (Model E10-10), each housed within isolation cubicles (Model 10-20), were used. Each cubicle utilized baffled air intake exhaust systems and ventilation fans. Each test cage contained three horizontally aligned, translucent plastic keys positioned approximately at a pigeon's standing sight line. Behind each key was a projector that displayed red, green, and yellow colors, as well as a white circle, onto a frosted rear projection screen (Coulbourn Model E21-18). Directly below the center key was a $5.7 \times 5 \mathrm{~cm}$ opening that, during reinforcement, provided access to a hopper containing mixed grain Within the opening was a lamp (Coulbourn Model E14-10 with bulb S11819X) that was illuminated only during reinforcement. Located $6.5 \mathrm{~cm}$ above the center key was a houselight that directed light upward to reflect light from the top of the cage (Coulbourn Model 14-10). The organization of all experimental events and recording of response choices was performed by a microcomputer system located in the same room. 
Procedure. Pigeons that had received previous training with either a dark ITI or an illuminated ITI in Santi et al. (2006) were all given 45 sessions of baseline training with a dark ITI. An individual trial commenced with a sample presentation in which the hopper light flashed on and off throughout a sequence. Each sequence began and ended with a light flash. The remainder of the flashes occurred at equally spaced time intervals between the initial and final flashes. The sample presentation always occurred in a darkened chamber (i.e., the hopper light flash was the only source of illumination during the sample presentation period). After the final flash of a sample sequence, a 1 -sec delay interval occurred. It was randomly either dark or illuminated by presentation of the houselight. The sample sequences used in Experiment 1 contained two few-sample sequences and two manysample sequences. One few sample (Few1) consisted of two 400msec flashes in $4 \mathrm{sec}$, and the second few sample (Few2) consisted of two $800-\mathrm{msec}$ flashes in $4 \mathrm{sec}$. For the many-sample sequences, one (Many1) consisted of four 200-msec flashes in $4 \mathrm{sec}$, and the second (Many2) consisted of four 400-msec flashes in $4 \mathrm{sec}$. Red was correct following the few-sample trials, whereas green was correct following the many-sample trials. A single peck to the comparison stimuli turned them off and if correct permitted 4-sec access to mixed grain from the illuminated food hopper. Incorrect responses to the comparison stimuli resulted in a 4-sec blackout followed immediately by the presentation of the same sample sequence, a 1-sec delay interval, and comparison stimulus configuration. A correct response on a correction trial resulted in 4-sec access to mixed grain, but only the choice response on the initial presentation of the trial was used to calculate matching accuracy. Within each block of eight trials, all combinations of the four sample stimuli, with comparison stimuli counterbalanced on the left and right side keys, occurred once. The order of presentation was randomized individually for each bird. The ITI, spent in darkness, was $15 \mathrm{sec}$, and each session consisted of 160 trials.

Delay testing was conducted for 20 sessions of 160 trials each. Within each session, 24 trials for each of the four samples (Few1, Few2, Many1, and Many2) occurred at the 1-sec baseline delay, and 4 trials for each sample occurred at each of the other delays $(0,2,4$, and $8 \mathrm{sec}$ ). For one half of the trials involving the 1-, 2-, 4-, and 8-sec delays, the entire delay was spent in darkness. On the other half of these trials, the houselight was illuminated for the entire delay. During delay testing, the correction procedure remained in effect only for 1-sec delay trials. Errors on all other delay trials were followed by a dark ITI and the presentation of the next sample sequence. All other parameters remained the same as those described previously. In all the statistical analyses reported in this article, the rejection region was $p<.05$.

\section{Results and Discussion}

Mean percentage of correct responding during the last two sessions of baseline training on 1-sec dark delay trials was $83.4 \%$ for Few $1,80.1 \%$ for Many $1,78.1 \%$ for Few2, and $84.8 \%$ for Many 2 . On the 1-sec illuminated delay trials, accuracy was $79.4 \%$ for Few1, 71.6\% for Many1, $77.6 \%$ for Few2, and $79.9 \%$ for Many 2 .

Mean percentage of correct responding during delay testing is shown in Figure 1. The top panel presents the data for trials with dark delay intervals, and the bottom panel presents the data for trials with illuminated delay intervals. During delay testing, one half of all 0-sec delay intervals were randomly designated as dark delays, with the other half randomly designated as illuminated delays. As expected, baseline training with a 1-sec dark and a 1 -sec illuminated baseline delay eliminated any response bias at these training delays. However, at extended dark delay intervals, a robust choose-few effect was observed, whereas at extended illuminated delays, there was no evidence of any response bias.


Figure 1. The mean percent of correct responses on few- and many-sample trials during delay test sessions following training with a 1-sec dark and 1-sec illuminated delay interval in Experiment 1 . The data for test trials with dark delays are shown in the top panel, and the data for test trials in which the delay interval was illuminated by the houselight are presented in the bottom panel. The values in parentheses following each sample type in the legend indicate the duration of the flash and the duration of the gap for that sample. Error bars represent the standard errors of the means.

An ANOVA was conducted on the delay test data with sample (few, many), set (set1, set2), delay interval illumination (dark, light), and delay interval length $(0,1,2,4$, and 8 $\mathrm{sec})$ as within-subjects factors. There were significant main effects of delay interval illumination $[F(1,7)=41.33]$ and delay interval length $[F(4,28)=39.45]$. There were significant two-way interactions of sample $\times$ delay interval length $[F(4,28)=4.36]$ and delay interval illumination $\times$ delay interval length $[F(4,28)=2.87]$. Finally, there was a significant three-way interaction of sample $\times$ delay interval illumination $\times$ delay interval length $[F(4,28)=5.38]$.

A simple main effects analysis of the three-way interaction indicated that there was a significant sample $\times$ delay interval length interaction only when the delay interval was dark $[F(4,28)=6.27]$. At the 8 -sec dark delay, accuracy was significantly higher for few samples than for many samples $[F(1,7)=14.15]$. When the delay interval was illuminated, only the main effect for delay interval length was significant $[F(4,28)=30.23]$. 
The retention functions obtained with dark delay intervals replicate those reported by Santi et al. (2006). There was no response bias at the 1-sec dark baseline delay interval, but at extended dark delays there was a significant choose-few effect. With illuminated delays, there was no evidence of a significant response bias at any delay interval. The absence of any bias with an illuminated delay suggests either that the small choose-few bias previously reported by Santi et al. (2006) is not a replicable effect, or that it was eliminated by baseline training with a constant 1 -sec illuminated delay. What is more important is that the overall pattern of results is quite robust and consistent with the instructional ambiguity explanation advanced by Santi et al. (2006). According to this account, pigeons respond on the basis of the similarity of the delay interval to the gap between light flashes. Long dark delays are confused with the longer dark gaps between flashes of the few samples, resulting in a response to the few-associated comparison stimulus. Illuminated delays are less similar to the dark gaps between flashes, so instructional ambiguity was reduced and no significant response bias was observed.

\section{EXPERIMENT 2}

If the previous interpretation of these results is correct, then it would be worthwhile to examine sequences of light flashes that control for the length of the gap between flashes so that the only reliable cue for responding to the comparisons is the number of flashes or the number of gaps. Table 2 shows the eight sample sequences used in Experiment 2. The top four sequences (GDV samples) were the same as those used in Experiment 1. The bottom four sequences were those that were added in Experiment 2 . These samples will be referred to as sequence duration varying (SDV) samples, because the total duration of these sequences differed by at least $6,400 \mathrm{msec}$ between the few and the many samples. The SDV few and many samples also differed in gap duration and sum of the gap durations, but the direction of the gap duration difference was opposite to that of the GDV few and many samples.

Data presented in Santi et al. (2006) indicated that multiple features of the light flash sequences control choice responding. The results of Experiment 1 suggested that gap duration was one of these features. If pigeons strongly rely on gap duration to discriminate the flash sequences, then when the SDV few and many samples were initially introduced in Experiment 2, accuracy for these samples should be below chance. That is, for the SDV few samples, the gap duration was the same as it was for the previously trained GDV many samples, and, as a result, a tendency to respond many would be anticipated. For the SDV many samples, the gap duration was the same as it was for the previously trained GDV few samples and as a result a tendency to respond few would be expected.

The most reliable discriminative cues for responding correctly to all eight sample sequences would be to rely on the number of flashes or the number of gaps. Temporal cues such as total sequence duration, flash duration, sum of the flash durations, sum of the gap durations, and gap between flashes were not consistently reliable across all eight of the sample sequences. Delay testing was conducted to determine whether retention functions differed from those previously obtained now that only the number of flashes or the number of gaps were consistently reliable cues for responding to comparison stimuli.

\section{Method}

Subjects and Apparatus. The subjects and apparatus used in Experiment 1 were also used in Experiment 2.

Procedure. After delay testing in Experiment 1, the pigeons were given two baseline training sessions with the four GDV samples. In the next session, the four SDV samples were introduced, and pigeons received training with all eight samples. The eight sample sequences controlled for the total sequence duration, the flash duration, the sum of the flash durations, the sum of the gap durations, and the gap between flashes (see Table 2). Within each session of 160 trials, the 1-sec dark and 1-sec illuminated delay intervals continued to be presented an equal number of times, and a correction procedure identical to that previously described was used. All other aspects of the training sessions were the same as described for Experiment 1. A total of 50 sessions of training were given with the four GDV and four SDV samples.

After training, all pigeons received delay testing for 20 sessions of 160 trials each. Within each session, 6 trials for each of the four GDV and four SDV samples (Few1, Few2, Many1, Many2) occurred at the 1-sec dark baseline delay, and 6 trials for each of the four GDV and four SDV samples (Few1, Few2, Many1, Many2) occurred at the 1 -sec illuminated baseline delay. There were 2 trials for each sample at each of the other delays $(0,2,4$, and $8 \mathrm{sec})$. For the 2-, 4-, and 8 -sec delays, half were spent in darkness, and half were illuminated by the houselight. During delay testing, the correction procedure remained in effect only for 1-sec delay trials. All other parameters remained the same as those described previously.

\section{Results and Discussion}

During the first five sessions of training with the GDV and SDV sequences, accuracy on the GDV samples remained well above chance and fairly stable after introduction of the SDV samples. However, accuracy on the SDV samples remained consistently at or slightly above chance over the five sessions following their introduction. This result is somewhat surprising, because if pigeons relied only on the gap between flashes to discriminate the sequences, then accuracy should have initially been below chance on the SDV sequences. Thus, pigeons appear to rely on more than just gap duration to discriminate sequences of light flashes.

Accuracy averaged over the last two training sessions on 1-sec dark delay trials for the GDV sequences was $71.8 \%, 74.1 \%, 76.5 \%$, and $72.8 \%$ on the Few1, Few2, Many1, and Many2 samples, respectively. On the 1-sec illuminated delay trials, accuracy for the GDV sequences was $65.6 \%, 77.5 \%, 69.9 \%$, and $63.9 \%$ on the Few1, Few2, Many1, and Many2 samples, respectively. During training on the novel SDV sequences, accuracy slowly improved over the 50 training sessions. Accuracy averaged over the last two training sessions on 1-sec dark delay trials for the SDV sequences was $70.4 \%, 79.4 \%, 77.1 \%$, and $75.6 \%$ on the Few1, Few2, Many1, and Many2 samples, respectively. On the 1-sec illuminated delay trials, accuracy for the SDV sequences was $66.3 \%, 71.6 \%, 65.0 \%$, and $71.4 \%$ on the Few1, Few2, Many1, and Many2 samples, respectively. 
Although accuracy was significantly above chance for all sample types, the moderate levels of accuracy and the length of training required to achieve them indicate that these discriminations were very difficult for the pigeons.

The delay testing results are presented in Figure 2. The top panel illustrates the data for trials with dark delay intervals, and the bottom panel illustrates the data for trials with illuminated delay intervals. During delay testing, one half of all 0-sec delay intervals were randomly designated as dark delays and the other half were randomly designated as illuminated delays. The data for Few1, Few2, Many1, and Many2 samples that are shown in Figure 2 were collapsed across the GDV and SDV sequences, because an analysis of the data revealed no significant differences between these sequences. As can be seen in Figure 2, when the delay interval was dark, the response biases obtained were very different from those previously observed in Experiment 1 or in Santi et al. (2006). Instead of a choose-few bias at extended dark delays, there was a marked choose-many bias. In addition, at the 0 -sec delay, the opposite response bias occurred; that is, pigeons responded more accurately following the few sample than following the many sample. In Experiment 1, no significant bias was evident at the 0 -sec delay, even though the baseline training delay for both experiments was the same. Retention functions in the illuminated delay condition did not show any response biases at extended delays. This result is the same as that in Experiment 1.

An ANOVA was conducted on the delay test data with sample (few, many), sequence type (GDV, SDV), set (set1, set2), delay interval illumination (dark, light), and delay interval length as within-subjects factors. There were significant main effects of delay interval illumination $[F(1,7)=$ $15.22]$ and delay interval length $[F(4,28)=25.02]$. There were significant two-way interactions of sample $\times$ delay interval illumination $[F(1,7)=15.19]$, sample $\times$ delay interval length $[F(4,28)=17.87]$, and delay interval illumination $\times$ delay interval length $[F(4,28)=7.92]$. There was a significant three-way interaction of sample $\times$ delay interval illumination $\times$ delay interval length $[F(4,28)=$ 5.55]. Finally, there was a significant four-way interaction of sample $\times$ set $\times$ delay interval illumination $\times$ delay interval length $[F(4,28)=4.02]$.

A simple main effects analysis of the four-way interaction indicated that when the delay interval was dark, there was a significant two-way interaction of sample $\times$ delay interval length $[F(4,28)=22.28]$. For the few samples, accuracy significantly decreased as the delay interval was lengthened $[F(4,28)=25.02]$. For the many samples, accuracy significantly increased as the delay interval was lengthened $[F(4,28)=10.38]$. In addition, accuracy was significantly greater for few samples than for many samples at the 0 -sec delay $[F(1,7)=90.53]$, but it was significantly greater for many samples than for few samples at both the 4 - and 8-sec delays $[F(1,7)=12.15$ and 22.50 , respectively $]$.

When the delay interval was illuminated, there was a significant two-way interaction of sample $\times$ delay interval length $[F(4,28)=4.03]$. Accuracy was significantly higher for few samples than for many samples at delays of $0 \mathrm{sec}$ $[F(1,7)=15.93]$ and $1 \mathrm{sec}[F(1,7)=26.40]$. However,
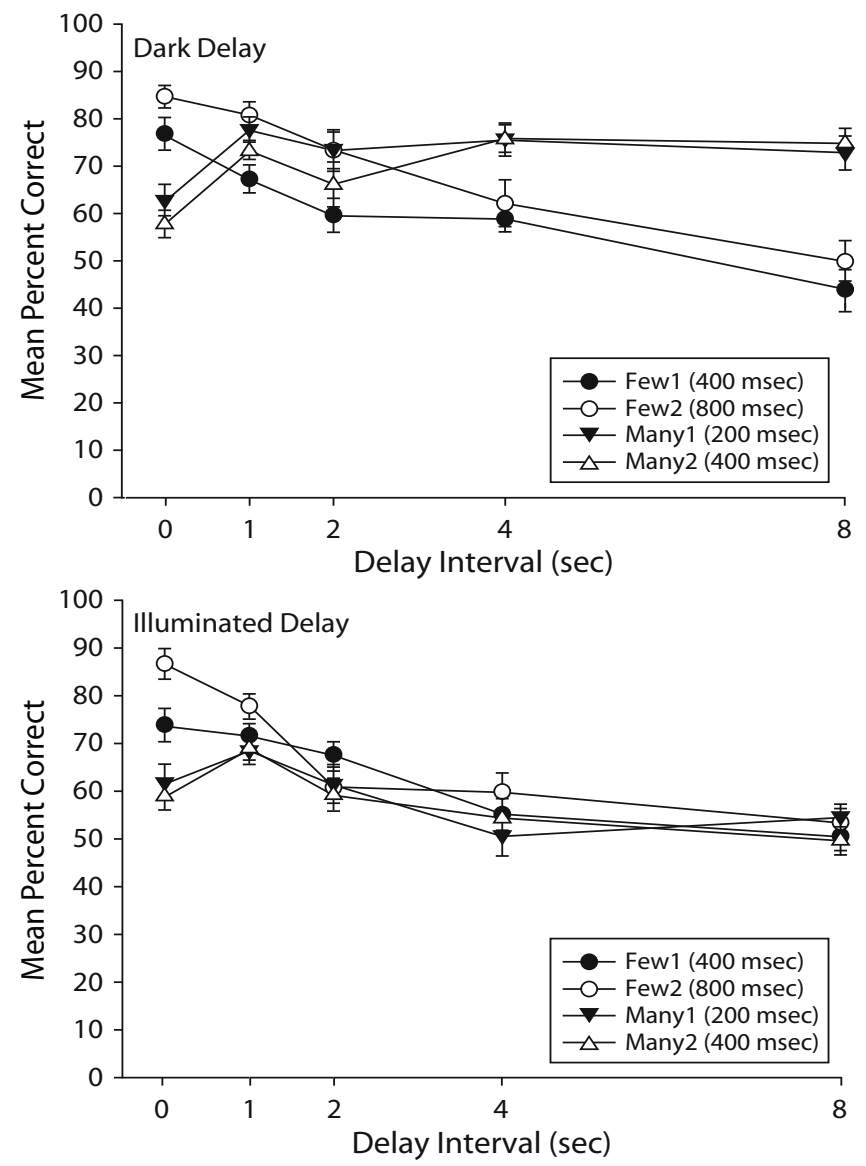

Figure 2. The mean percent of correct responses on the fewand many-sample trials during delay testing collapsed across the GDV and the SDV sequences in Experiment 2. The data for test trials with dark delays are shown in the top panel, and the data for test trials in which the delay was illuminated by the houselight are presented in the bottom panel. The values in parentheses following each sample type in the legend indicate the duration of the flash for that sample. Error bars represent the standard errors of the means.

there was no significant difference in accuracy for few and many samples at the 2-, 4-, and 8-sec illuminated delays.

The present experiment provided better control for temporal features of the light flash sequences so that only the number of flashes and the number of gaps were consistently reliable cues for responding to comparison stimuli. If pigeons had encoded either number of flashes or number of gaps as pulse counts resulting from the operation of an event switch, and if pulse counts had been gradually lost during a delay interval, then one would have expected a choose-few bias at extended delays regardless of whether the delays were dark or illuminated. Not only was the choose-many response bias opposite to that expected, but it was evident only at extended dark delays. Just as for the findings reported in Santi et al. (2006), it seems that the ambiguity hypothesis may provide the most plausible explanation. Trials with extended dark delay intervals may have been confused with samples that had very long dark gaps. Note that to control for the gap between flashes as a discriminative cue, the present experiment introduced four additional sample 
sequences with very short sequence durations in the case of few samples, and very long sequence durations in the case of many samples. During baseline training, trials with a very long interval (greater than $4 \mathrm{sec}$ ) between the first flash and the occurrence of comparison stimuli were more likely to be trials for which the many response was correct. Long extended dark delays may have been confused with the many samples that had relatively long sequence durations $(8,800$ or $10,400 \mathrm{msec})$ and three long dark gaps of 2,400 or 3,200 msec. This confusion would have resulted in a choose-many response bias at long dark delays. When the extended delays were illuminated by the houselight, this response bias did not occur, presumably because illuminated delays were more discriminably different than the dark delays from the light flash sequences.

Interestingly, when the comparison stimuli were presented immediately after the last flash of a sequence $(0-$ sec delay), there was a significant choose-few bias. This could have occurred if a novel 0 -sec delay made the last flash seem longer in duration than it would have seemed following a 1-sec delay. Trials with the longest flash duration were few-sample trials. Although the confusion interpretation placed on these data is clearly post hoc, it is evident that these results provided additional evidence that pigeons rely on multiple temporal features rather than number to discriminate between different sequences of light flashes.

Although a reduction in ambiguity between the sample and delay appears to eliminate response biases, it does not appear to reduce the overall number of errors. That is, overall accuracy did not appear to be improved when the delay was illuminated rather than dark. Thus, as a reviewer of an earlier version of this article suggested, one could view these findings in terms of generalization decrement rather than instructional ambiguity. However, it is not clear that generalization decrement provides a better explanation of the failure to obtain higher accuracy when the delay is illuminated than the ambiguity hypothesis does.

\section{GENERAL DISCUSSION}

In Experiment 1, pigeons were trained to discriminate between sequences of two and four flashes of feeder light with a 1-sec dark and 1-sec houselight-illuminated delay interval. The samples sequences controlled for the total sequence duration, the duration of an individual flash, the sum of the flash durations, and the sum of the gap durations. The number of flashes, the number of gaps, and the duration of the gap between the flashes were the only reliable discriminative cues. Retention functions with a dark delay interval revealed a significant choose-few bias at the 8 -sec delay. On the other hand, retention functions with an illuminated delay interval yielded no biased forgetting. The delay functions in Experiment 1 were consistent with those reported in Santi et al. (2006). They found a significant choose-few bias at 4 and $8 \mathrm{sec}$ when the delay interval was dark, and a modest choose-few bias when the delay interval was illuminated. Both sets of results suggest that pigeons utilized the duration of the gap between flashes to discriminate few and many samples. Long dark delays were confused with the long dark gap between flashes of the few samples, and they resulted in a bias to respond to the few comparison. Illuminated delays were less similar than dark delays to the dark gaps between flashes, so instructional ambiguity did not occur and no significant response biases were obtained.

In Experiment 2, additional sample sequences were added so that the gap between light flashes also became an unreliable cue for discriminating the few and many samples. Only the number of flashes or the number of gaps could be used reliably to discriminate all of the sample sequences. On the first session of training, accuracy was at chance on the novel SDV samples, but it remained above chance on the previously trained GDV samples. If pigeons were relying on the duration of the gap between flashes to discriminate few from many, one would have anticipated that accuracy would have been significantly below chance on the novel SDV samples. This result suggests that pigeons utilized more than just gap duration to discriminate few from many samples, and it reinforces the conclusion reached in Santi et al. (2006) that multiple features of these sequential events are used as the basis for responding to comparison stimuli.

During delay testing in Experiment 2, pigeons exhibited a choose-few bias at the 0 -sec delay interval, and a choose-many bias at extended dark delay intervals. No bias was observed at extended illuminated delay intervals. The choose-many bias was surprising, because training pigeons with a procedure that controlled for all temporal features might have been expected to produce a reliance on number. According to the subjective shortening hypothesis, a choose-few bias would have been predicted at extended delay intervals. It is difficult to provide an entirely satisfactory explanation for the retention functions obtained in Experiment 2. For example, one explanation for the choose-many bias observed at extended dark delay intervals could be that pigeons sometimes relied on the total sequence duration to make their response choices. The total sequence duration for the SDV many samples was either $10,400 \mathrm{msec}$ (Many1) or $8,800 \mathrm{msec}$ (Many2), whereas for the SDV few samples it was either $1,867 \mathrm{msec}$ (Few1) or 2,400 msec (Few2). Relying on the total sequence duration would be useful in discriminating between SDV samples. During delay testing, a trial with a long delay might appear to be more similar to an SDV many sample than to an SDV few sample, because there would be a long time interval between the first flash on a trial and the presentation of comparisons. As a result, a pigeon might be more likely to respond to the manyassociated comparison stimulus on trials with long delays. However, there are some problems with this explanation. First, all GDV samples (few and many) were 4,000 msec in duration, making the total sequence duration an unreliable cue for these samples. Hence, if pigeons relied only on the total sequence duration, they would not be able to respond accurately following a GDV sample. Since accuracy to GDV samples was well above chance, this suggests that pigeons relied on more than just the total sequence duration to discriminate between the sequences. However, even if pigeons used the total sequence duration only when it was useful (i.e., on SDV samples), why did the choose-many 
bias occur only following extended dark delays and not following extended illuminated delays? In order to deal with this result, one would need to assume that only the extended dark delays were perceived as similar to the SDV many samples, whereas the extended illuminated delays were not. Although this may not be an entirely satisfactory explanation of the retention functions, it is clear that pigeons appear to rely on multiple features of the sequences to make discriminative responses, and that stimulus conditions during the delay interval can be similar to these discriminative features and result in biased responding.

Overall, these experiments make several contributions to understanding how pigeons discriminate and remember sequences of light flashes. First, pigeons rely on temporal information if it is available to them. Second, pigeons utilize multiple features of a sequence rather than rely on a single feature. Third, instructional ambiguity appears to be the best explanation of the biased forgetting effects observed when memory for light flash sequences is tested.

\section{AUTHOR NOTE}

This research was supported by Grant OGPOOD6378 from the Natural Sciences and Engineering Research Council of Canada to A.S. The authors thank Kelley Putzu and Kristin Lukashal for their animal care assistance. The data reported in this article were part of an MSc thesis submitted by the first author to Wilfrid Laurier University. Correspondence concerning this article should be addressed to A. Santi, Department of Psychology, Wilfrid Laurier University, Waterloo, ON, N2L 3C5 Canada (e-mail: asanti@wlu.ca).

\section{REFERENCES}

Dorrance, B. R., Kaiser, D. H., \& Zentall, T. R. (2000). Eventduration discrimination by pigeons: The choose-short effect may result from retention-test novelty. Animal Learning \& Behavior, 28, 344-353.

Fetterman, J. G. (1995). The psychophysics of remembered duration. Animal Learning \& Behavior, 23, 49-62.

Fetterman, J. G. (2000). Biases in pigeon working memory. Animal Learning \& Behavior, 28, 68-79.

Fetterman, J. G., \& MacEwen, D. (1989). Short-term memory for responses: The "choose-small" effect. Journal of the Experimental Analysis of Behavior, 52, 311-324.

Gaitan, S. C., \& Wixted, J. T. (2000). The role of "nothing" in memory for event duration in pigeons. Animal Learning \& Behavior, 28, 147-161.

Grant, D. S. (1993). Coding processes in pigeons. In T. R. Zentall (Ed.), Animal cognition: A tribute to Donald A. Riley (pp. 193-216). Hillsdale, NJ: Erlbaum.

GRANT, D. S. (2006). Training delays reduce the choose-short effect with keylight duration samples in pigeons. Behavioural Processes, 72, 139-148.

Grant, D. S., \& Kelly, R. (1996). The role of minimum wait time and sample discriminability in the coding of event duration in pigeons. Learning \& Motivation, 27, 243-259.

Grant, D. S., \& Kelly, R. (1998). The effect of variable-delay training on coding of event duration in pigeons. Learning \& Motivation, 29, 49-67.

Grant, D. S., \& Spetch, M. L. (1991). Pigeons' memory for event duration: Differences between choice and successive matching tasks. Learning \& Motivation, 22, 180-199.

Grant, D. S., \& Spetch, M. L. (1993). Analogical and nonanalogical coding of samples differing in duration in a choice-matching task in pigeons. Journal of Experimental Psychology: Animal Behavior Processes, 19, 15-25.

Grant, D. S., \& Spetch, M. L. (1994). The role of asymmetrical coding of duration samples in producing the choose-short effect in pigeons. Learning \& Motivation, 25, 413-430.

Grant, D. S., Spetch, M. L., \& Kelly, R. (1997). Pigeons' coding of event duration in delayed matching-to-sample. In C. M. Bradshaw \& E. Szabadi (Eds.), Time and behaviour: Psychological and neurobehavioural analyses (pp. 217-264). Amsterdam: Elsevier.

Hope, C., \& SANTI, A. (2004). Pigeons' memory for number of events: Effects of intertrial interval and delay interval illumination. Learning \& Motivation, 35, 348-370.

Kelly, R., \& Spetch, M. L. (2000). Choice biases in delayed matchingto-sample duration with pigeons: Manipulations of ITI and delay illumination. Quarterly Journal of Experimental Psychology, 53B, 309-323.

Kraemer, P. J., Mazmanian, D. S., \& Roberts, W. A. (1985). The choose-short effect in pigeon memory for stimulus duration: Subjective shortening versus coding models. Animal Learning \& Behavior, 13, 349-354.

Meck, W. H., \& Church, R. M. (1983). A mode control model of counting and timing process. Journal of Experimental Psychology: Animal Behavior Processes, 9, 320-334.

Roberts, W. A., Macuda, T., \& Brodbeck, D. R. (1995). Memory for number of light flashes in the pigeon. Animal Learning \& Behavior, 23, 182-188.

Roberts, W. A., \& Mitchell, S. (1994). Can a pigeon simultaneously process temporal and numerical information? Journal of Experimental Psychology: Animal Behavior Processes, 20, 66-78.

Santi, A., Bridson, S., \& Ducharme, M. J. (1993). Memory codes for temporal and nontemporal samples in many-to-one matching by pigeons. Animal Learning \& Behavior, 21, 120-130.

Santi, A., Ducharme, M. J., \& Bridson, S. (1992). Differential outcome expectancies and memory for temporal and nontemporal stimuli in pigeons. Learning \& Motivation, 23, 156-169.

SANTI, A., \& Hope, C. (2001). Errors in pigeons' memory for number of events. Animal Learning \& Behavior, 29, 208-220.

Santi, A., Lellwitz, J., \& Gagne, S. (2006). Pigeons' memory for sequences of light flashes: Reliance on temporal properties and evidence for delay interval/gap confusion. Behavioural Processes, 72, $128-138$.

Sherburne, L. M., Zentall, T. R., \& Kaiser, D. H. (1998). Timing in pigeons: The choose-short effect may result from pigeons" "confusion" between delay and intertrial intervals. Psychonomic Bulletin \& Review, 5, 516-522.

Spetch, M. L. (1987). Systematic errors in pigeons' memory for event duration: Interaction between training and test delay. Animal Learning \& Behavior, 15, 1-5.

Spetch, M. L., \& RusaK, B. (1989). Pigeons' memory for event duration: Intertrial interval and delay effects. Animal Learning \& Behavior, 17, 147-156.

Spetch, M. L., \& RusaK, B. (1992). Temporal context effects in pigeons' memory for event duration. Learning \& Motivation, 23, 117-144.

Spetch, M. L., \& Wilkie, D. M. (1983). Subjective shortening: A model of pigeons' memory for event durations. Journal of Experimental Psychology: Animal Behavior Processes, 9, 14-30.

Zentall, T. R. (1997). Animal memory: The role of "instructions." Learning \& Motivation, 28, 280-308.

ZENTALL, T. R. (1999). Support for a theory of memory for event duration must distinguish between test trial ambiguity and actual memory loss. Journal of the Experimental Analysis of Behavior, 72, 467-472.

ZENTALL, T. R. (2005). Timing, memory for intervals, and memory for untimed stimuli: The role of instructional ambiguity. Behavioural Processes, 70, 209-222.

(Manuscript received July 18, 2006; revision accepted for publication December 14, 2006.) 